\title{
PSI-Guided Mandible-First Orthognathic Surgery: Maxillo-Mandibular Position Accuracy and Vertical Dimension Adjustability.
}

\section{Giovanni Badiali}

IRCCS Azienda Ospedaliero-Universitaria di Bologna, Via Albertoni 15, Bologna, Italy.

Mirko Bevini ( $\square$ mirko.bevini@studio.unibo.it)

Department of Biomedical and Neuromotor Sciences, University of Bologna, Italy.

Ottavia Lunari

University of Bologna

\section{Elisa Lovero}

Department of Biomedical and Neuromotor Sciences, University of Bologna, Italy.

\section{Federica Ruggiero}

IRCCS Azienda Ospedaliero-Universitaria di Bologna, Via Albertoni 15, Bologna, Italy.

\section{Federico Bolognesi}

IRCCS Azienda Ospedaliero-Universitaria di Bologna, Via Albertoni 15, Bologna, Italy.

\section{Liliana Feraboli}

IRCCS Azienda Ospedaliero-Universitaria di Bologna, Via Albertoni 15, Bologna, Italy.

\section{Alberto Bianchi}

Maxillofacial Surgery Unit, Dept. of Surgery and Surgical Specialties, Azienda Ospedaliero Universitaria

"Rodolico - S. Marco", University of Catania, Italy.

\section{Claudio Marchetti}

IRCCS Azienda Ospedaliero-Universitaria di Bologna, Via Albertoni 15, Bologna, Italy.

\section{Research Article}

Keywords: orthognathic, BSSO, PSIs

Posted Date: January 18th, 2021

DOI: https://doi.org/10.21203/rs.3.rs-145748/v1

License: (c) (1) This work is licensed under a Creative Commons Attribution 4.0 International License.

Read Full License 
Version of Record: A version of this preprint was published at Journal of Personalized Medicine on November 21st, 2021. See the published version at https://doi.org/10.3390/jpm11111237. 


\section{PSI-guided mandible-first orthognathic surgery: maxillo- mandibular position accuracy and vertical dimension adjustability.}

Giovanni Badiali, M.D. Ph.D. ${ }^{1,2}$, Assistant Professor of Oral and Maxillofacial Surgery, Department of Biomedical and Neuromotor Sciences, University of Bologna (Italy). Email: giovanni.badiali@unibo.it

Mirko Bevini, M.D. ${ }^{2 *}$ Research Fellow, Department of Biomedical and Neuromotor Sciences, University of Bologna (Italy).

Email: mirko.bevini@studio.unibo.it

Ottavia Lunari, D.D.S. ${ }^{3}$, M.D. Student, University of Bologna (Italy).

Email: ottavia.lunari@studio.unibo.it

Elisa Lovero, D.D.S. ${ }^{2}$, Ph.D. Student, Department of Biomedical and Neuromotor Sciences, University of Bologna (Italy). Email: elisa.lovero@unibo.it

Federica Ruggiero, M.D. ${ }^{1,2}$, Ph.D. Student, Department of Biomedical and Neuromotor Sciences, University of Bologna (Italy) Email: ruggierof.md@gmail.com

Federico Bolognesi, M.D. ${ }^{1,2}$, Ph.D. Student, Department of Biomedical and Neuromotor Sciences, University of Bologna (Italy). Email: federico.bolognesi5@unibo.it

Liliana Feraboli, M.D. ${ }^{2}$, Resident in Oral and Maxillofacial Surgery, University of Bologna (Italy). Email: ferabolililiana@gmail.com

Alberto Bianchi, M.D., D.D.S, Ph.D. ${ }^{4}$, Full Professor of Oral and Maxillofacial Surgery, University of Catania (Italy). Email: alberto.bianchi@unict.it

Claudio Marchetti, M.D., D.D.S. 1, 2, Full Professor of Oral and Maxillofacial Surgery, Department of Biomedical and Neuromotor Sciences, University of Bologna (Italy). Email: claudio.marchetti@unibo.it

\section{Affiliations}

1) IRCCS Azienda Ospedaliero-Universitaria di Bologna, Via Albertoni 15, Bologna, Italy.

2) Department of Biomedical and Neuromotor Sciences, University of Bologna, Italy.

3) University of Bologna, Italy.

4) Maxillofacial Surgery Unit, Dept. of Surgery and Surgical Specialties, Azienda Ospedaliero Universitaria "Rodolico - S. Marco", University of Catania, Italy.

* - Corresponding author 


\begin{abstract}
In orthognathic surgery, patient-specific osteosynthesis implants (PSIs) represent a novel approach for the reproduction of the virtual surgical planning on the patient.

The aim of this study is to analyse the quality of maxillo-mandibular positioning using a hybrid mandible-first mandibular-PSI guided procedure on twenty-two patients while the upper maxilla was fixed using manually-bent stock titanium miniplates.

The virtual surgical plan was used to guide the design of PSIs and positioning guides, which were then 3D printed using biocompatible materials. A CBCT scan was performed one month after surgery and postoperative facial skeletal models were segmented for comparison against the surgical plan. A three-dimensional cephalometric analysis was carried out on both planned and obtained anatomies. A Spearman correlation matrix was computed on the calculated discrepancies, in order to achieve a more comprehensive description of maxillo-mandibular displacement.

Intraoperatively, all PSIs were successfully applied. The procedure was found to be accurate in planned maxillo-mandibular positioning reproduction, while maintaining a degree of flexibility to allow for aesthetics-based verticality correction in a pitch range between -5.31 and $+1.79 \mathrm{~mm}$. Such correction did not significantly affect the achievement of planned frontal symmetry.
\end{abstract}




\section{Introduction}

The mandible-first approach for orthognathic surgery is an alternative procedure to the more widespread maxilla-first approach, which carries an array of theoretical advantages, such as reducing the mandibular condylar sag and reducing the strain on the upper maxillary miniplates while carrying out the mandibular BSSO, subsequently improving the quality of planning reproduction ${ }^{1}$.

While part of the advantages has not been demonstrated, the increased intraoperative flexibility offered by this approach in terms of vertical correction is counterbalanced by its reliance on the exactness of the post-osteosynthesis spatial relationship between proximal and distal mandibular segments. In the pursuit of a solution to such issue, we combined the mandible-first approach with a PSI-guided mandibular procedure and demonstrated that it leads to a satisfactory reproduction of the planned mandibular anatomy ${ }^{2}$. Current literature on mandibular and bimaxillary PSI-guided orthognathic surgery is still poor, and the potential failure due to dental interferences caused by combined maxillary and mandibular inaccuracies beyond the surgeon's level of control, has been reported but has not been extensively investigated yet ${ }^{3,4}$.

We determined the surgically achieved positional accuracy of both the upper maxilla and mandibular teeth-bearing fragment, in relation to the cranial base, on a cohort of patients treated with this approach. In this paper we evaluate the precision and flexibility of the approach itself, in terms of aesthetics-based intraoperative verticality correction, also assessing whether such correction could lead to other unwanted displacements of the skeletal segments analysed. We also considered the frontal symmetry and the yaw component of asymmetries, which is the most difficult to control in traditional splint-guided surgery ${ }^{5}$.

\section{Materials and Methods}

Twenty-two patients undergoing orthognathic bimaxillary surgery at the Oral and Maxillofacial Surgery Unit of Sant'Orsola-Malpighi University Hospital (Bologna, Italy) between July 2017 and June 2019 - seven males and fifteen females, mean age 26 years (range 18-43 years) - were included in the trial. Eight patients were diagnosed with skeletal class II deformity (one with combined facial asymmetry), ten were diagnosed with skeletal class III (six with combined asymmetry), three patients were diagnosed with class I facial asymmetry and one with anterior open bite. The present protocol was approved by the Sant'Orsola-Malpighi University Hospital ethics committee (approval number 238/2012/0/Disp PL02, amended 18/10/2016); the study conformed to the principles of the Declaration of Helsinki. Written informed consent was obtained from all the patients upon enrollment in the trial ${ }^{2}$.

Case Planning and Surgery

One month prior to surgery all patients underwent a pre-operative CBCT-scan (NewTom VGI Evo, Cefla Group, Imola, Italy), $(24 \times 19 \mathrm{~cm}$ FOV, $0.3 \mathrm{~mm}$ voxel $)$ in a clinically-determined natural head position and using a wax bite obtained in clinically-set condylar centric relation. Contextually, dental digital models were acquired using the CS 3600 intraoral scanner (Carestream Health Inc, Rochester, NY, USA), and 3D-printed using a stereolithographic printer 
(Form 2, Formlabs Inc., Somerville, MA, USA). The final occlusion was determined on the stereolithographic models and transferred to the digital models via re-scan ${ }^{2}$.

IPS Case Designer software (KLS Martin, Tuttlingen, Germany) was used to perform a threedimensional cephalometry according to Swennen et al ${ }^{6}$ and plan the skeletal movements (Fig. 1A). Proximal and distal mandibular segments were positioned in order to reduce interference along the osteosynthesis surface and minimize discontinuity of the inferior border.

Intermediate and final surgical splints were designed according to the virtual surgical plan. Although the mandibular procedure was designed as a potentially splint-less surgery, intermediate surgical splints were manufactured as a back-up solution in case of intraoperative failure of the system. Eventually, the intermediate splint was systematically used to stabilize the teeth-bearing fragment during fixation.

On the basis of the VSP, KLS Martin biomedical engineers designed the individualized mandibular-PSI positioning guides, patient-specific plates, and splints under the surgeon's suggestions (Fig. 1B). The positioning guides were designed to guide the buccal and sagittal osteotomies, and the cranio-caudal level of the lingual osteotomy to reproduce the digitally designed Bilateral Sagittal Split Osteotomy (BSSO). Screw trajectories were planned in order to avoid dental roots and the path of the inferior alveolar nerve (Fig. 1B). Three guide types were used throughout the trial and were compared in our previous work on this cohort of patients ${ }^{2}$. The custom 3D-printed titanium alloy (Ti6Al4V) plates were designed to fixate the proximal and distal mandibular fragments in their planned positions using the screw fixation holes, drilled according to the positioning guides, as reference. Splints were manufactured using 3D printed dental resin. Digital three-dimensional models of bony fragments and plates were also provided by KLS Martin (in .STL format) for outcome evaluation.

GB operated all patients by means of mandible-first approach, using the PSI system (Figure 2B, C). The mandibular bony surface was exposed through the conventional vestibular incision to perform BSSO. Two titanium screws were used to secure the guide to the mandible using the designed fixation holes $(1.5 \mathrm{~mm})$ to avoid mobilization while performing the the osteotomy. According to the guide, the surgeon marked the osteotomy lines using ultrasonic bone-cutting tools (Piezo-Surgery, Mectron, Cerasco, Italy) and drilled the transfer holes for the plate using an Angulus2 angulated drill (KLS Martin, Tuttlingen, Germany). The guide was then removed to complete the osteotomy. The condyle-bearing and the teeth-bearing fragments were fixed in the planned position using the pre-drilled transfer holes $(2.0 \mathrm{~mm})$ to position the PSIs. The intermediate splint was used-positioned to secure the mandibular teeth-bearing fragment in position while performing the osteosynthesis to facilitate the procedure. The upper maxilla was then fixed in the best aesthetics-based vertical position using standard manually-bent titanium miniplates and $2.0 \mathrm{~mm}$ screws, under the guide of the final splint (Fig. 2A).

Outcome analysis

A CBCT scan was performed on all patients one month after surgery, before any tooth movements occurred, thanks to rigid orthodontics and daily use of the final splint. The same machine and parameters of the pre-operative scan were used; the occlusion was kept at maximum intercuspation. The post-operative CBCT scan was segmented to obtain a 3D model of 
the post-operative skull, mandible, and mandibular plates using DICOM to Print software ( $3 D$ Systems, Rock Hill, SC, USA) and exported in STL format ${ }^{2}$.

Two analyses were carried out: cephalometric and rigid body transformation.

The positions of planned and post-operative .STL objects were compared using the open source software CloudCompare (CloudCompare Project, www.cloudcompare.org). In order to fix a frame of reference, the planned and obtained cranial base models were superimposed via an iterative closest point (ICP) alignment method (Fig. 3A). The alignment was then visually checked by means of colorimetric surface maps interface. The discrepancy between planned and obtained post-operative position of the mandibular teeth bearing fragment and upper maxilla were evaluated by analyzing the displacement in terms of rotation (roll, pitch and yaw angles) and translation (antero-posterior, lateral and vertical). These movements were determined aligning the planned model to the post-operative result via ICP alignment and colorimetric map inspection (Fig. 3B, C), then evaluating the translational shift and the rotation of the model according to Euler angles convention ${ }^{2}$.

The signed discrepancies were tabulated applying conventional signs. Positive signed values identify forward, upward and left lateral translations; a positive pitch angle identifies a clockwise rotation as seen from the patient's right lateral aspect; a positive roll angle identifies a clockwise rotation as seen from in front of the patient; a positive yaw angle indicates a clockwise rotation as seen caudally to the patient.

Two further parameters were defined to comprehensively describe the angular and translational displacement of each 3D model considered: total angular error and total translational error. The first is the angle in the axis-angle representation of a rigid body transformation, while the second is the module of the translation vector. Both measures are always positive by definition and were used to avoid positive and negative displacements canceling each other on average in the description of displacement ${ }^{2}$.

A three-dimensional cephalometric analysis was performed on planned and postoperative 3D models. The considered cephalometric data is shown in Table 1

Statistical Analyses

All measures were summarized using median and interquartile range (IQR), due to the limited sample size $(\mathrm{n}=22)$. However, to allow for comparison with the existing literature, mean and standard deviation were also provided for each measure.

In order to assess how the intraoperative surgical plan correction impacted on the symmetry if the result and overall precision, a Spearman correlation matrix $(n=22)$ was calculated for each considered rotation, translation, and cephalometric plan to post-op difference. Rotational and translational discrepancies between VSP and postoperative results were considered both as signed and absolute values.

Non-parametric two-tailed tests were used in all cases. The significance level was set to $\alpha=0.05$ for all tests. IBM SPSS Statistics 25 (IBM, Armonk, NY, USA) was used to perform the analyses. 


\section{Results}

The average follow-up is 22 months (range 13-37). Descriptive statistics are reported in Tables 2 and 3.

In the maxillary segment positioning, we obtained a median total angular error of $2.31^{\circ}$ (IQR $1.41^{\circ}$ ) and a median total translational error of $2.31 \mathrm{~mm}$ (IQR $1.83 \mathrm{~mm}$ ). With the exception of the pitch angle (median absolute value $1.51^{\circ}$ ), the alteration of which is compatible with the intraoperative verticality correction based on the autorotation of the mandible, median absolute value rotational shifts were around $0.75^{\circ}$ from planned (roll $0.78^{\circ}$, yaw $0.72^{\circ}$ ). Translational median discrepancies were all within $1.5 \mathrm{~mm}$ in absolute value from planned. The greatest discrepancy can be found in anteroposterior translation, with a median of $1.39 \mathrm{~mm}$ in absolute value, and a tendency to retrusion, with a signed median value of $-1.27 \mathrm{~mm}$.

In the mandibular teeth bearing fragment positioning, the median total angular error was $2.34^{\circ}$ $\left(\mathrm{IQR} 1.30^{\circ}\right)$ and the median total translational error was $2.01 \mathrm{~mm}$ (IQR $1.34 \mathrm{~mm}$ ). Similarly to the upper maxilla, the median pitch angle was $1.2^{\circ}$ in absolute value, with yaw and roll both $0.75^{\circ}$ in absolute value. Median absolute value translations were also around $1 \mathrm{~mm}$ (lateral $=$ $0.93 \mathrm{~mm}$, antero-posterior $=1 \mathrm{~mm}$, vertical $=0.70 \mathrm{~mm}$ )

Median maxillary and mandibular roto-translations obtained are simulated on an example case and a colorimetric surface map between planned and simulated shifted position was calculated; The result is shown in Fig. 4.

The frontal symmetry cephalometric measures considered in absolute value yielded median results below $1.5 \mathrm{~mm}$ on midline measures (A/Sag, B/Sag, Pog/Sag, UIs/Sag and LIs/Sag) and on the $\Delta \mathrm{Go} / \mathrm{sag}$. The largest median discrepancy was obtained in the $\Delta \mathrm{U} 3 / \mathrm{Sag}$ at $2.78 \mathrm{~mm}$, while the median $\Delta \mathrm{U} 6 / \mathrm{Sag}$ was $1.65 \mathrm{~mm}$.

Spearman's correlation coefficients deemed clinically significant were extrapolated from the correlation matrix and reported in Table 4.

All following reference to cephalometric measurements is to be intended as difference between planned and post-op values.

Strong positive correlations were found between analogous translational and rotational values of upper maxilla and mandibular teeth-bearing fragment, ranging from 0.523 with $p=0.012$ for pitch to 0.819 with $\mathrm{p}<0.001$ for anteroposterior translation.

A-McNamara and Incisal Protrusion are negatively correlated $(-0.519, p=0,013)$ as expected in a situation in which antero-posterior discrepancy is partly due to intraoperative modification of the verticality. This finding is also supported by the correlation between mandibular pitch and maxillary antero-posterior translation $(-0.542, \mathrm{p}=0.009)$ Notably, no significant correlations were found between symmetry cephalometric indicators (Maxillary Deviation, Mandibular Deviation, Mental Deviation, UIs/Sagittal Plane, Lis/Sagittal plane, U3/Sagittal Plane and U6/Sagittal Plane) and pitch or vertical translation. 


\section{Discussion}

The debate between maxilla-first and mandible-first approach has been ongoing for decades and, although the maxilla-first approach is more widely used, the mandible-first approach is preferable in a range of cases, for the fact that it provides a more stable frame of reference by using the upper maxilla as a guide. Also, executing the BSSO first avoids the stress caused on the miniplates used for maxillary fixation, and theoretically reduces the condylar displacement caused by the supine position of the patient and anesthesia. However, as Borba et al. state in their systematic review on the matter, little data is available to support these claims ${ }^{1}$.

Although the use of PSIs in maxillary surgery is already well documented ${ }^{7,8,9,10}$, their use in mandibular orthognathic surgery is less frequently reported, mainly due to the difficulty of obtaining a stable reference for plate fixation on the lateral mandibular aspect ${ }^{3,4,11,12}$. Suojanen et al. ${ }^{4}$, in a cohort study of 30 patients, reported a significant percentage of cases in which PSIs were inapplicable or needed modification. More recent studies, however, do not report this occurrence, although examining smaller cohorts of patients.

Li et al. ${ }^{3}$ reported satisfactory precision results obtained on a cohort of patients using bimaxillary PSIs $(n=10)$ : this procedure requires minimal to no planning error with flawless subsequent execution of said plan, and allows no intraoperative plan correction, potentially resulting in skeletal and dental interferences, thus rendering PSI application unfeasible. Furthermore, the rigidity and relative brittleness of sintered titanium alloy makes the modification of custom-made implants almost impossible. Double jaw PSI solutions are also significantly more expensive than single jaw PSIs, a factor affecting the adoption of this approach.

Published data on PSI-guided mandibular orthognathic surgery suggests that the approach we are hereby presenting has never been proposed before by other authors. As per our previous findings on this cohort of patients ${ }^{4}$, this procedure yielded an accurate mandibular anatomy reproduction, although needing a more thorough planning -when compared to empiric proximal fragment positioning- to avoid interferences between the bony segments along the BSSO osteosynthesis surface; interferences in this region could in turn lead to condylar displacement in the glenoid fossa.

From a clinical point of view, the aesthetic result was positive in all patients, part of which is due to the intraoperative plan correction in which the modification of soft tissue could be accounted for in real time. Plate infections $(4.5 \%)$ are on par with the rate observed in patients in which conventional titanium plates were used ${ }^{13}$.

The overall accuracy of the procedure, also taking into account the vertical correction, is highly satisfactory. Although the median absolute value mandibular autorotation was only $1.51^{\circ}$, giving a median verticality correction of $0.93 \mathrm{~mm}$ in absolute value, no correlation between frontal symmetry shift measures and pitch or vertical translation was found in an autorotation range between $-4.03^{\circ}$ and $+2.24^{\circ}$, allowing for a vertical plan correction between $-5.31 \mathrm{~mm}$ and +1.79 $\mathrm{mm}$. 
Our interpretation of the obtained maxillary translational values suggests that a quota of anteroposterior shift (median $-1.27 \mathrm{~mm}$ ) could partly be a byproduct of the verticality correction via autorotation (mandibular pitch and maxillary antero-posterior translation are correlated with a coefficient of -0.542 and $p=0.009$ ) and partly to be attributed to a quota of condylar sag or displacement in the fossa, due to the supine position of the patient and myorelaxation. This displacement, however measurable, was not clinically significant and did not impact on the resulting occlusion.

The correlation values according to which the upper maxilla and the mandibular teeth-bearing fragment moved in a coordinated fashion further the notion of accuracy of CAD-CAM splints, in this case mainly the final splint.

\section{Conclusion}

Following our previous work on the matter, the findings above support the hypothesis that the proposed mandible-first mandibular PSI-guided procedure yields a positive outcome without sacrificing adjustabilty and thus reducing the risk of PSI inapplicability. Vertical intraoperative plan correction through mandibular autorotation does not correlate with midline shift, allowing for a safe vertical correction ranging between -5.31 and $+1.79 \mathrm{~mm}$, although in most cases there was no need for intraoperative plan correction, and the plan was accurately transferred to the patient as previously designed. Notably, this procedure achieved an accurate correction of the yaw component of asymmetries, the least controllable in splint-guided surgery. All in all, the adoption of single jaw PSIs could be considered a viable and more economically sustainable alterative to double jaw PSIs. 


\section{References}

1. Borba, A. M. et al. Mandible-first sequence in bimaxillary orthognathic surgery: A systematic review. International Journal of Oral and Maxillofacial Surgery 45, 472-475 (2016).

2. Badiali, G., Bevini, M., Ruggiero, F. et al. Validation of a patient-specific system for mandible-first bimaxillary surgery: ramus and implant positioning precision assessment and guide design comparison. Sci Rep 10, 13317 (2020).

3. Li, B. et al. A new approach of splint-less orthognathic surgery using a personalized orthognathic surgical guide system: A preliminary study. International Journal of Oral and Maxillofacial Surgery 46, 1298-1305 (2017).

4. Suojanen, J., Leikola, J. \& Stoor, P. The use of patient-specific implants in orthognathic surgery: A series of 30 mandible sagittal split osteotomy patients. Journal of CranioMaxillofacial Surgery 45, 990-994 (2017)

5. Tominaga $\mathrm{K}$, Habu M, Tsurushima H, Takahashi O, Yoshioka I. CAD/CAM splint based on soft tissue 3D simulation for treatment of facial asymmetry. Maxillofac Plast Reconstr Surg. 2016 Jan 27;38(1):4. doi: 10.1186/s40902-016-0050-8

6. Swennen, G. R. J., Schutyser, F., Hausamen, J. E. \& Van Cleynenbreugel, J. Threedimensional cephalometry: A color atlas and manual. Three-Dimensional Cephalometry: A Color Atlas and Manual 1-365 (Springer Berlin Heidelberg, 2006). doi:10.1007/3-54029011-7

7. Mazzoni, S., Bianchi, A., Schiariti, G., Badiali, G. \& Marchetti, C. Computer-aided design and computer-aided manufacturing cutting guides and customized titanium plates are useful in upper maxilla waferless repositioning. Journal of Oral and Maxillofacial Surgery 73, 701-707 (2015).

8. Kraeima, J., Jansma, J. \& Schepers, R. H. Splintless surgery: does patient-specific CADCAM osteosynthesis improve accuracy of Le Fort I osteotomy? British Journal of Oral and Maxillofacial Surgery 54, 1085-1089 (2016).

9. Suojanen, J., Leikola, J. \& Stoor, P. The use of patient-specific implants in orthognathic surgery: A series of 32 maxillary osteotomy patients. Journal of Cranio-Maxillofacial Surgery 44, 1913-1916 (2016). 
10. Xue, C. et al. Precise control of maxillary multidirectional movement in Le Fort I osteotomy using a surgical guiding device. British Journal of Oral and Maxillofacial Surgery 56, 797-804 (2018).

11. Savoldelli, C., Vandersteen, C., ... Santini, J., 2018. Dental occlusal-surface-supported titanium guide to assist cutting and drilling in mandibular bilateral sagittal split osteotomy. Journal of Stomatology, Oral and Maxillofacial Surgery 119, 75-78.

12. Brunso, J. et al. Custom-Machined Miniplates and Bone-Supported Guides for Orthognathic Surgery: A New Surgical Procedure. Journal of Oral and Maxillofacial Surgery 74, 1061.e1-1061.e12 (2016).

13. O'Connell, J., Murphy, C., Ikeagwuani, O., Adley, C. \& Kearns, G. The fate of titanium miniplates and screws used in maxillofacial surgery: $A 10$ year retrospective study. International Journal of Oral and Maxillofacial Surgery 38, 731-735 (2009).

\section{Authors Contribution:}

Study Conception: G.B., A.B., C.M.; Study Design: G.B., M.B.; Data Collection and Analysis: M.B., E.L., O.L., F.R., F.B., L.F.; Data Interpretation: G.B., M.B., E.L. O.L., Manuscript Drafting: M.B., G.B., O.L., F.R., Figures and Photography: M.B., E.L.

\section{Figure Legends}

\section{Figure 1}

Figure 1A: Virtual surgery planning in IPS CaseDesigner.

Figure 1B: Plates CAD. Teeth roots and inferior alveolar nerves were segmented and carefully avoided while planning the plates' position and screw trajectories.

\section{Figure 2}

Intraoperative view of maxillary manually bent plates (2A), mandibular positioning guide (2B), and mandibular CAD/CAM plate (2C).

\section{Figure 3}

Analysis method in CloudCompare software. Planned models (orange) were aligned to postoperative models (blue) on the basis of the cranial base (3A). Planned upper maxilla was aligned to postoperative maxillary position and the transformation was recorded; the alignment was visually checked via generation of colorimetric surface maps (3B); An analogous protocol was applied to the mandibular teeth-bearing fragment (3C). 


\section{Figure 4}

Simulation of the obtained median maxillary and mandibular displacements on an example surgical plan in CloudCompare. A colorimetric surface map was used to show surface differences of the simulated median position from planning. Rami were not included in the simulation in order to increase the visibility of the teeth-bearing fragment. An analogous simulation for the rami position relative to the teeth-bearing fragment is shown in our previous work on this cohort ${ }^{2}$. 


\section{Table 1}

Considered cephalometric measurements and relative description.

\begin{tabular}{|c|c|}
\hline \multicolumn{2}{|c|}{ Considered cephalometric measurements } \\
\hline A/Sag & Distance of point A from the Sagittal Plane (Maxillary Deviation) \\
\hline B/Sag & $\begin{array}{l}\text { Distance of point B from the Sagittal Plane (Mandibular } \\
\text { Deviation). }\end{array}$ \\
\hline Pog/Sag & $\begin{array}{l}\text { Distance of Pogonion from the Sagittal Plane (Mental } \\
\text { Deviation). }\end{array}$ \\
\hline Uls/Sag & Distance from Upper Incisors midpoint to Sagittal plane. \\
\hline Lls/Sag & $\begin{array}{l}\text { Distance in millimeters from Lower Incisors midpoint to Sagittal } \\
\text { plane. }\end{array}$ \\
\hline$\Delta \mathrm{Go} / \mathrm{Sag}$ & $\begin{array}{l}\text { Difference between left and right Gonion to Sagittal plane } \\
\text { distances in millimeters. }\end{array}$ \\
\hline$\Delta$ U3/Sag & $\begin{array}{l}\text { Difference between left and right Upper canine to Sagittal plane } \\
\text { distances. }\end{array}$ \\
\hline$\Delta$ U6/Sag & $\begin{array}{l}\text { Difference between left and right first Upper molars to Sagittal } \\
\text { plane distances. }\end{array}$ \\
\hline A/McNamara & Distance of point A from McNamara plane. \\
\hline Incisal Protrusion & $\begin{array}{l}\text { Distance in millimeters of the Upper Incisors midpoint to plane } \\
\text { A (plane parallel to McNamara plane passing though point A). }\end{array}$ \\
\hline
\end{tabular}




\section{Table 2}

Descriptive statistics of rigid body transformation analysis results. Measures in absolute value are indicated as 'abs.'.

\begin{tabular}{|c|c|c|c|c|c|c|c|c|c|c|c|c|c|c|c|}
\hline & & Pitch & $\begin{array}{l}\text { Pitch } \\
\text { abs. }\end{array}$ & Roll & $\begin{array}{l}\text { Roll } \\
\text { abs. }\end{array}$ & Yaw & $\begin{array}{l}\text { Yaw } \\
\text { abs. }\end{array}$ & $\begin{array}{l}\text { Tot. Ang. } \\
\text { Displ. }\end{array}$ & Lateral & $\begin{array}{l}\text { Lat. } \\
\text { abs. }\end{array}$ & $\begin{array}{l}\text { Ant.- } \\
\text { Post. }\end{array}$ & $\begin{array}{l}\text { A-P } \\
\text { abs. }\end{array}$ & Vertical & $\begin{array}{l}\text { Vert. } \\
\text { abs. }\end{array}$ & $\begin{array}{l}\text { Total } \\
\text { trans. }\end{array}$ \\
\hline \multirow{4}{*}{ Maxilla } & Average & -0.90 & 1.54 & -0.06 & 0.93 & -0.02 & 0.91 & 2.33 & -0.08 & 1.05 & -1.45 & 1.70 & -0.78 & 1.14 & 2.58 \\
\hline & St.Dev. & 1.65 & 1.06 & 1.21 & 0.75 & 1.20 & 0.76 & 0.91 & 1.28 & 0.70 & 1.87 & 1.64 & 1.37 & 1.08 & 1.70 \\
\hline & Median & -0.92 & 1.52 & 0.00 & 0.78 & -0.28 & 0.72 & 2.31 & -0.23 & 0.99 & -1.27 & 1.39 & -0.78 & 0.93 & 2.03 \\
\hline & IQR & 2.50 & 1.53 & 1.55 & 0.60 & 1.50 & 0.69 & 1.41 & 1.86 & 0.99 & 2.46 & 2.38 & 1.07 & 0.77 & 1.83 \\
\hline \multirow{4}{*}{ Mandible } & Average & 0.83 & 1.53 & 0.39 & 0.96 & 0.73 & 1.13 & 2.55 & -0.32 & 1.14 & -1.26 & 1.49 & -0.61 & 1.05 & 2.51 \\
\hline & St.Dev. & 1.88 & 1.34 & 1.24 & 0.85 & 1.33 & 0.99 & 1.20 & 1.50 & 1.00 & 1.78 & 1.59 & 1.51 & 1.23 & 1.81 \\
\hline & Median & 0.36 & 1.20 & 0.46 & 0.75 & 0.41 & 0.75 & 2.34 & -0.18 & 0.93 & -0.84 & 0.99 & -0.26 & 0.71 & 2.02 \\
\hline & IQR & 2.59 & 1.52 & 1.04 & 0.72 & 2.00 & 1.38 & 1.30 & 1.54 & 0.91 & 1.41 & 1.18 & 1.05 & 1.20 & 1.35 \\
\hline
\end{tabular}




\section{Table 3}

Descriptive statistics of cephalometric analysis results. Measures in absolute value are indicated as 'abs.'.

\begin{tabular}{|c|c|c|c|c|c|c|c|c|c|c|c|c|c|c|c|c|}
\hline & A/Sag & $\begin{array}{l}\text { A/Sag } \\
\text { abs. }\end{array}$ & B/Sag & $\begin{array}{l}\text { B/Sag } \\
\text { abs. }\end{array}$ & Pog/Sag & $\begin{array}{l}\text { Pog/Sag } \\
\text { abs. }\end{array}$ & $\begin{array}{c}\Delta \\
\text { Go/Sag }\end{array}$ & $\begin{array}{c}\Delta \\
\text { Go/Sag } \\
\text { abs. }\end{array}$ & Uls/Sag & $\begin{array}{l}\text { Uls/Sag } \\
\text { abs }\end{array}$ & Lls/Sag & $\begin{array}{l}\text { Lls/Sag } \\
\text { abs }\end{array}$ & $\begin{array}{c}\Delta \\
\text { U3/Sag }\end{array}$ & $\begin{array}{c}\Delta \\
\text { U3/Sag } \\
\text { abs }\end{array}$ & $\begin{array}{c}\Delta \\
\text { U6/Sag }\end{array}$ & $\begin{array}{c}\Delta \\
\text { U6/Sag } \\
\text { abs }\end{array}$ \\
\hline Average & 0.52 & 0.96 & 0.86 & 1.31 & 1.00 & 1.67 & -0.56 & 2.40 & 0.88 & 1.20 & 0.71 & 1.02 & 0.17 & 2.89 & -0.39 & 2.10 \\
\hline St.Dev. & 1.17 & 0.83 & 1.36 & 0.91 & 1.91 & 1.33 & 3.26 & 2.23 & 1.30 & 0.99 & 1.29 & 1.04 & 3.55 & 1.97 & 2.70 & 1.68 \\
\hline Median & 0.30 & 0.83 & 1.10 & 1.19 & 1.04 & 1.50 & -0.48 & 1.35 & 0.71 & 1.02 & 0.44 & 0.58 & 0.12 & 2.78 & -0.54 & 1.65 \\
\hline IQR & 1.30 & 1.15 & 1.41 & 1.12 & 2.38 & 2.16 & 2.65 & 2.90 & 1.30 & 1.01 & 0.82 & 1.01 & 5.40 & 3.58 & 3.09 & 1.85 \\
\hline
\end{tabular}




\section{Table 4}

Clinically significant Spearman's correlation coefficients extrapolated from the correlation matrix.

\begin{tabular}{|c|c|c|}
\hline Mx pitch & corr. coeff. & -0.008 \\
\hline A/Sag & Sig.2-tail & 0.970 \\
\hline Mx pitch & corr. coeff. & -0.221 \\
\hline B/Sag & Sig.2-tail & 0.323 \\
\hline Mx pitch & corr. coeff. & -0.317 \\
\hline Pog/Sag & Sig.2-tail & 0.151 \\
\hline Mx pitch & corr. coeff. & 0.018 \\
\hline$\Delta \mathrm{Go} / \mathrm{Sag}$ & Sig.2-tail & 0.938 \\
\hline Mx pitch & corr. coeff. & 0.318 \\
\hline UIs/Sag & Sig.2-tail & 0.149 \\
\hline Mx pitch & corr. coeff. & -0.053 \\
\hline Lis/Sag & Sig.2-tail & 0.816 \\
\hline Mx pitch & corr. coeff. & 0.220 \\
\hline$\Delta$ U3/Sag & Sig.2-tail & 0.326 \\
\hline Mx pitch & corr. coeff. & 0.307 \\
\hline$\Delta$ U6/Sag & Sig.2-tail & 0.165 \\
\hline Md pitch & corr. coeff. & 0.020 \\
\hline A/Sag & Sig.2-tail & 0.930 \\
\hline
\end{tabular}

\begin{tabular}{|c|c|c|}
\hline Md pitch & corr. coeff. & -0.037 \\
\hline B/Sag & Sig.2-tail & 0.871 \\
\hline Md pitch & corr. coeff. & -0.167 \\
\hline Pog/Sag & Sig.2-tail & 0.459 \\
\hline Md pitch & corr. coeff. & 0.084 \\
\hline$\Delta \mathrm{Go} / \mathrm{Sag}$ & Sig.2-tail & 0.710 \\
\hline Md pitch & corr. coeff. & 0.169 \\
\hline Uls/Sag & Sig.2-tail & 0.453 \\
\hline Md pitch & corr. coeff. & -0.109 \\
\hline Lis/Sag & Sig.2-tail & 0.629 \\
\hline Md pitch & corr. coeff. & -0.012 \\
\hline$\Delta$ U3/Sag & Sig.2-tail & 0.958 \\
\hline Md pitch & corr. coeff. & 0.047 \\
\hline$\Delta$ U6/Sag & Sig.2-tail & 0.836 \\
\hline Mx pitch & corr. coeff. & $.523^{*}$ \\
\hline Md pitch & Sig.2-tail & 0.012 \\
\hline Mx roll & corr. coeff. & $.729^{* *}$ \\
\hline Md roll & Sig.2-tail & 0.000 \\
\hline
\end{tabular}

\begin{tabular}{|c|c|c|}
\hline Mx yaw & corr. coeff. & $.613^{* *}$ \\
\hline Md yaw & Sig.2-tail & 0.002 \\
\hline Mx lat & corr. coeff. & $.581^{* *}$ \\
\hline Md lat & Sig.2-tail & 0.005 \\
\hline$M \times a-p$ & corr. coeff. & $.819^{* *}$ \\
\hline Md a-p & Sig.2-tail & 0.000 \\
\hline Mx vert & corr. coeff. & $.648^{* *}$ \\
\hline Md vert & Sig.2-tail & 0.001 \\
\hline Mx pitch & corr. coeff. & $-.461^{*}$ \\
\hline Md a-p & Sig.2-tail & 0.031 \\
\hline Mx pitch & corr. coeff. & $-.522^{*}$ \\
\hline Md vert & Sig.2-tail & 0.013 \\
\hline Md pitch & corr. coeff. & $-.542^{* *}$ \\
\hline$M \times A-P$ & Sig.2-tail & 0.009 \\
\hline Md pitch & corr. coeff. & $-.612^{* *}$ \\
\hline Mx vert & Sig.2-tail & 0.002 \\
\hline $\mathrm{A} / \mathrm{McN}$ & corr. coeff. & $-.519^{*}$ \\
\hline Inc. Prot. & Sig.2-tail & 0.013 \\
\hline
\end{tabular}




\section{Figures}
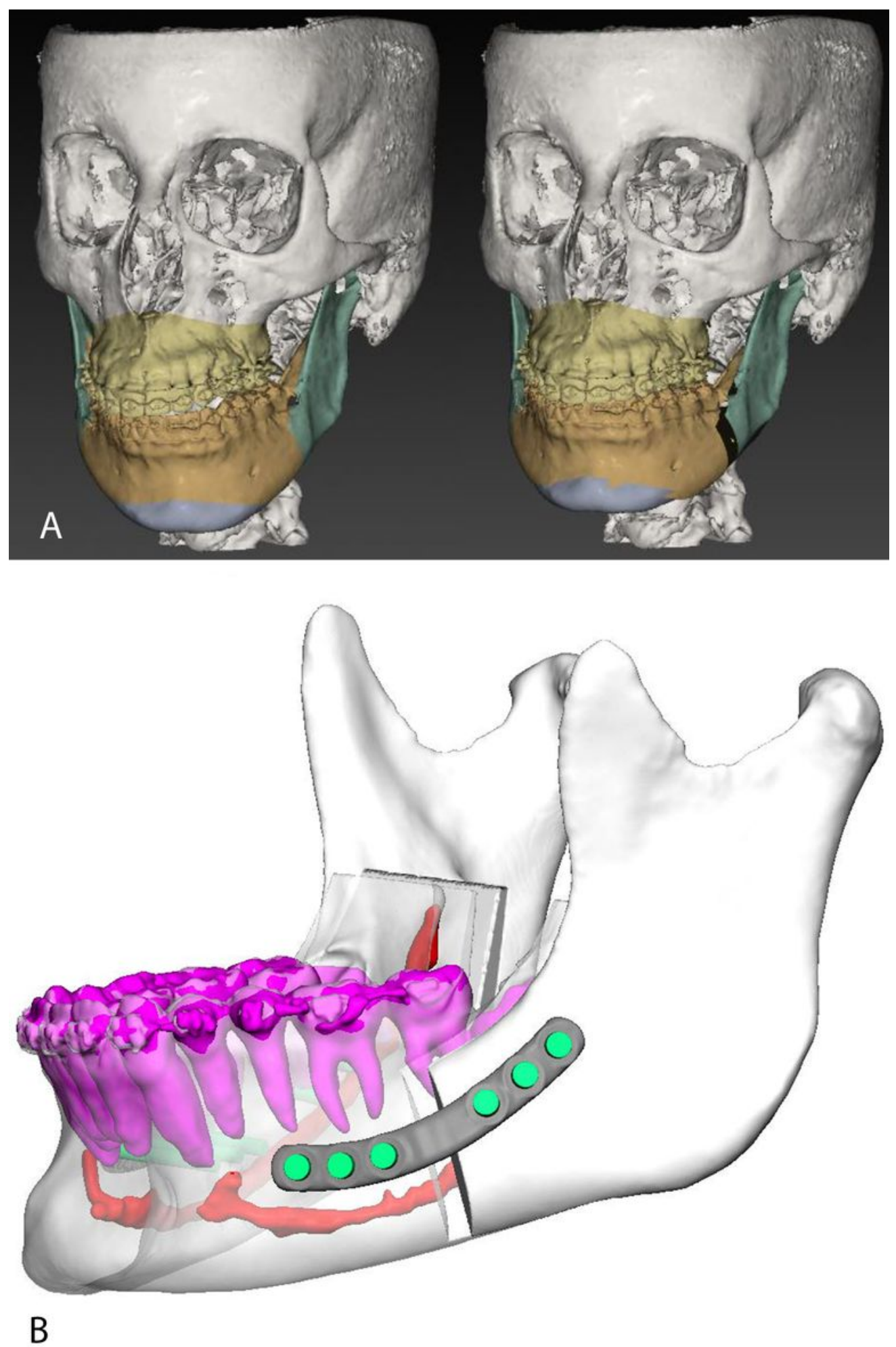

\section{Figure 1}

Figure 1A: Virtual surgery planning in IPS CaseDesigner. Figure 1B: Plates CAD. Teeth roots and inferior alveolar nerves were segmented and carefully avoided while planning the plates' position and screw trajectories. 

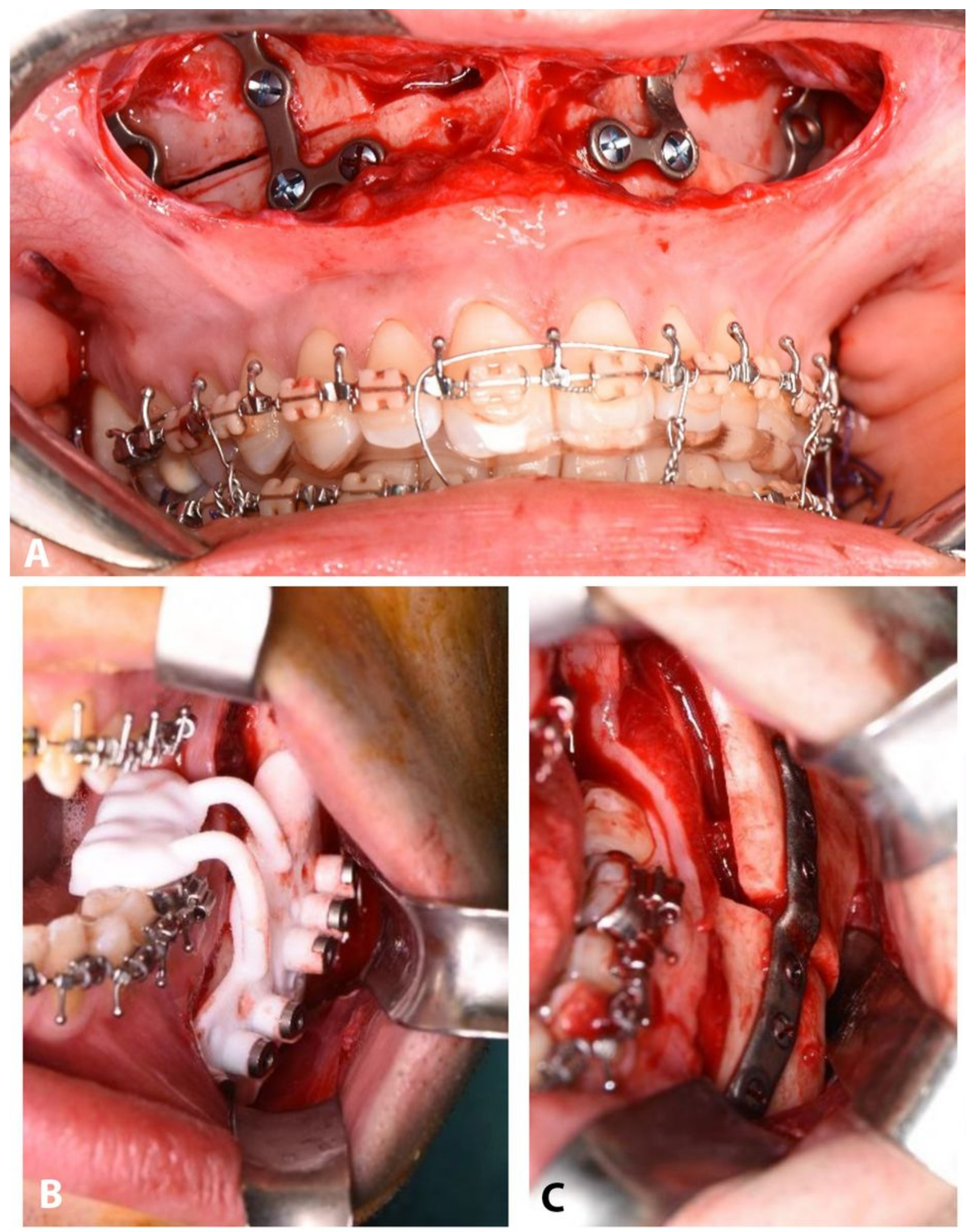

\section{Figure 2}

Intraoperative view of maxillary manually bent plates (2A), mandibular positioning guide (2B), and mandibular CAD/CAM plate (2C). 


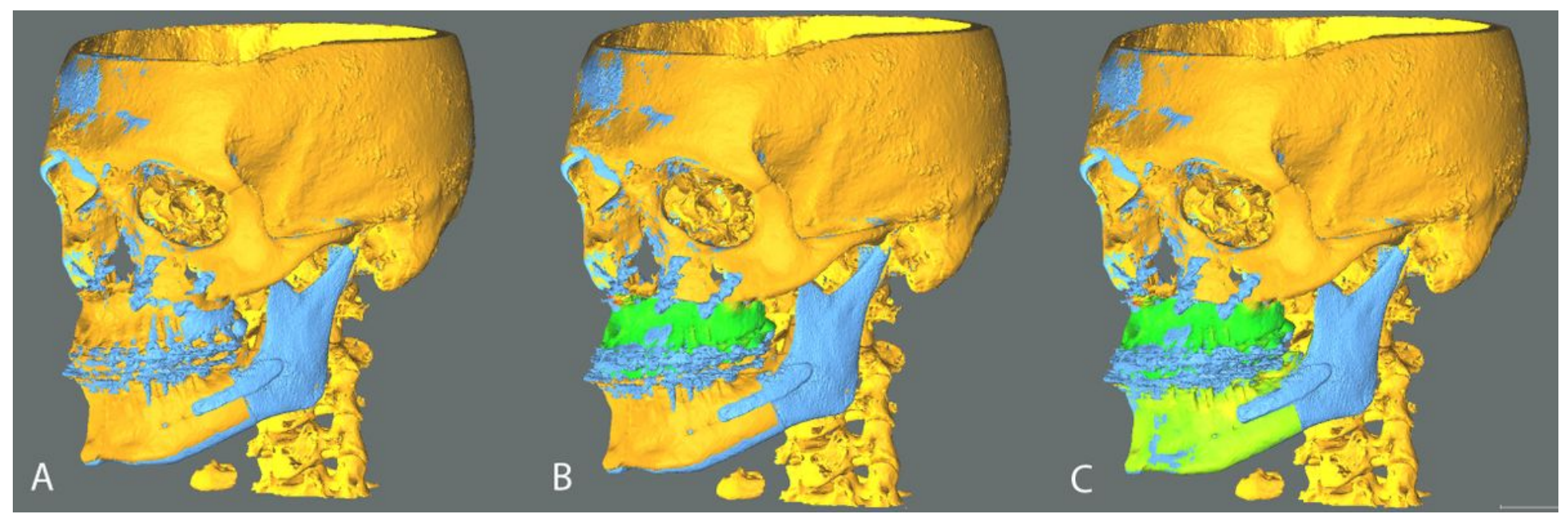

\section{Figure 3}

Analysis method in CloudCompare software. Planned models (orange) were aligned to postoperative models (blue) on the basis of the cranial base (3A). Planned upper maxilla was aligned to postoperative maxillary position and the transformation was recorded; the alignment was visually checked via generation of colorimetric surface maps (3B); An analogous protocol was applied to the mandibular teeth-bearing fragment (3C).

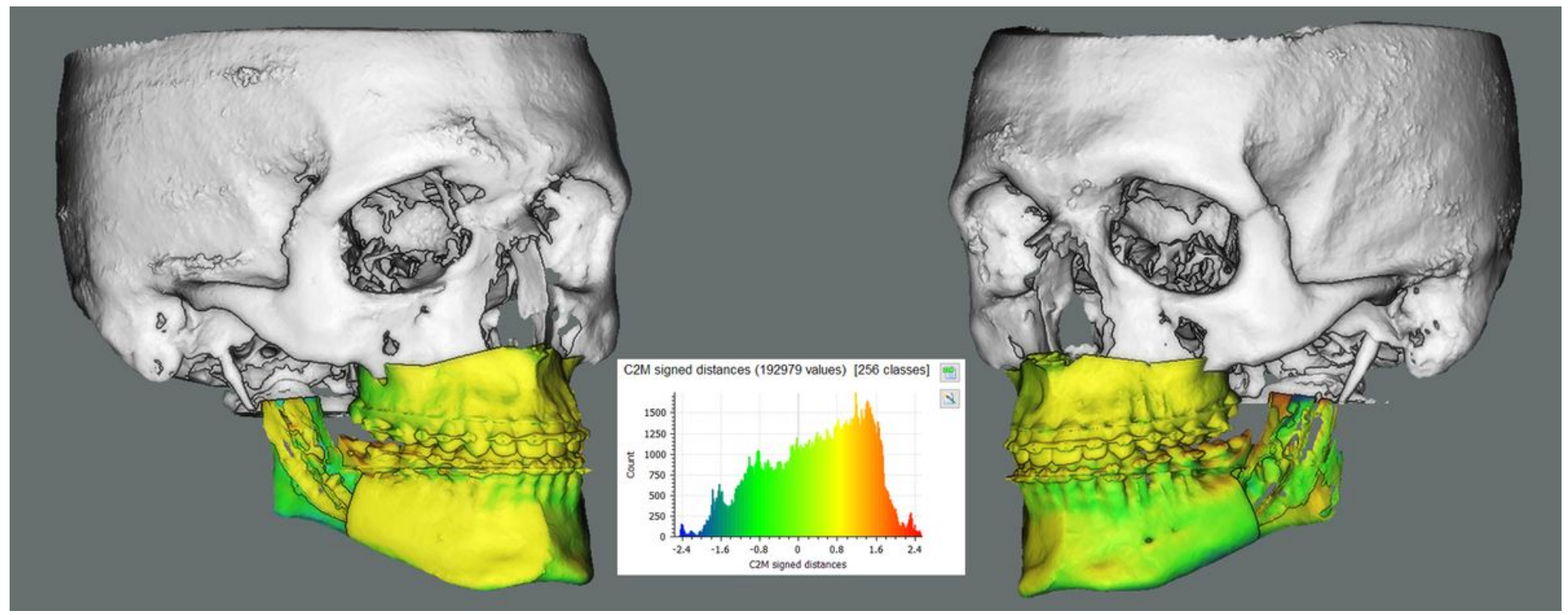

\section{Figure 4}

Simulation of the obtained median maxillary and mandibular displacements on an example surgical plan in CloudCompare. A colorimetric surface map was used to show surface differences of the simulated median position from planning. Rami were not included in the simulation in order to increase the visibility of the teeth-bearing fragment. An analogous simulation for the rami position relative to the teeth-bearing fragment is shown in our previous work on this cohort 2. 